\title{
Resting-State FMRI Single Subject Cortical Parcellation Based on Region Growing
}

\author{
Thomas Blumensath ${ }^{1}$, Timothy E.J. Behrens ${ }^{1,2}$, and Stephen M. Smith ${ }^{1}$ \\ 1 Oxford Centre for Functional MRI of the Brain (FMRIB Centre), \\ University of Oxford, Oxford, UK \\ \{tblumens, behrens, steve\}@fmrib.ox.ac.uk \\ WwW.fmrib.ox.ac.uk \\ 2 Welcome Trust Centre for Neuroimaging, 12 Queen Square, London, UK
}

\begin{abstract}
We propose a new method to parcellate the cerebral cortex based on spatial dependancy in the fluctuations observed with functional Magnetic Resonance Imaging (fMRI) during rest. Our surface-based approach uses a region growing method. In contrast to previous methods, locally stable seed points are identified on the cortical surface and these are grown into a (relatively large 1000 to 5000) number of spatially contiguous regions on both hemispheres. Spatially constrained hierarchical clustering is then used to further combine these regions in a hierarchical tree. Using short-TR resting state fMRI data, this approach allows a subject specific parcellation of the cortex into anatomically plausible subregions, identified with high scan-to-scan reproducibility and with borders that delineate clear changes in functional connectivity.
\end{abstract}

\section{Introduction}

A subdivision of the human cerebral cortex into anatomically and functionally distinct regions has long been a fundamental goal in the study of the human brain. Measuring Blood Oxygen Level Dependant (BOLD) signal fluctuations during rest using functional MRI (fMRI) techniques allows studies of such functional subdivisions in the living human brain. We propose a novel automated procedure to derive such a partition (or parcellation) for an individual subject using resting state BOLD data. Not only is this single subject approach able to uncover fundamental organisational principles, but is also anticipated to better reveal subject specific variations in human functional brain anatomy. Furthermore, the robust specification of cortical parcellations is a fundamental first step in the definition and study of functional brain networks [1, which model statistical dependencies between the parcel's characteristic time-courses.

\section{Methodology}

A wide range of statistical tools has been used over the years to derive cortical parcellations from resting state fMRI data. Methods include those based 
on ICA, spectral clustering, hierarchical clustering, k-means (or fuzzy k-means) clustering, mixture model based clustering and many more [234]. Region growing approaches for fMRI-based parcellation have previously been suggested in [567]. Our approach uses the following steps:

1. We use ICA based automatic de-noising of the data set.

2. We perform our analysis on the cortical surface.

3. We define a fairly large number of 'locally stable' seeds.

4. The seeds are then grown into spatially contiguous regions.

5. A cluster tree is build using spatially constrained hierarchical clustering.

\subsection{Subjects and Data Acquisition}

We used data from a previous study [8]. For each of the 5 subjects (ages 18-25, 3 males and 2 females) six 10-miniute fMRI datasets were acquired in a single session (eyes closed) using the accelerated protocol describe in [9], providing whole brain coverage at a TR of 0.8 seconds and with an isotropic spatial resolution of $3 \mathrm{~mm}$. Data was acquired on a 3 Tesla standard commercial scanner (Siemens Trio) equipped with $40 \mathrm{mT} / \mathrm{m}$ gradients with a slew rate of $200 \mathrm{mT} / \mathrm{m} / \mathrm{ms}$. In each scanning session, a single EPI reference image was acquired between each of the 10 minute blocks. A 1x1x1 $\mathrm{mm}^{3}$ resolution structural image (T1-weighted) was acquired to aid registration. One of the subjects was scanned 3 times, on separate days, so the full data-set consisted of 4210 minute sections. Of these, 36 were no-task resting-state paradigms (30 with eyes closed, 6 with eyes open). The remaining 6 scans (eyes open) used a silent backwards counting task. The imaging protocol used for human studies was approved by the institutional review board at the University of Minnesota. Each of the subjects provided informed written and verbal consent prior to participating in the research.

\subsection{Preprocessing}

FSL (FMRIB's Software Library) [10] was used for 1) head motion correction 2) full width 200s temporal high-pass filtering and 3) ICA de-noised with spatialICA using MELODIC [11. 4) T1 images were segmented using FreeSurfer [12] and the functional data was projected onto the cortical surface using FreeSurfer's mri_vol2surf functionality.

\subsection{Defining Locally Stable Seeds}

BOLD signal to noise ratio (SNR) is not uniform across the cortical surface. However, there are currently no methods that would allow us to estimate this varying SNR reliably. Nevertheless, measures used to assess functional connectivity will indicate a decreased connectivity if spatially independent noise is present, so that brain areas with higher noise contamination will in general show lower connectivity. To partially address the SNR problem, we therefore propose an approach that is based on the estimation of cortical locations that can be assumed to be less noisy. 
A further constraint we would like to impose on these locations is that they are placed well inside a functionally homogeneous brain region. We calculate a spatial map that encodes both of these properties. We here computed the local standard deviation in a $3 \mathrm{~mm}$ radius, that is we subtract the ROI's mean time course from each of the ROI's time-courses and then calculate the standard deviation of the concatenated demeaned time-courses. Larger ROI radii gave similar results (though this produced smoother maps and thus fewer seed points). Seed points were found to be distributed approximately uniformly over the cortex.

To further reduce the variance of the estimated map (at the expense of spatial resolution), we post-process the map using a surface based Gaussian kernel smoother with a full width half $\max$ of $2.35 \mathrm{~mm}(\sigma=1 \mathrm{~mm})$. Seed voxels are then found as the local minima within the smoothed map.

\subsection{Growing the Seeds}

Seeds are grown into different clusters using region growing. Pearson correlation is calculated between the seed's time-course and the time-courses of its neighbouring vertices. Neighbouring vertices are then joined to a seed's cluster if their similarity measure is within $90 \%$ of the maximal similarity found within this iteration. The $90 \%$ threshold was chosen to provide a good compromise between speed (higher thresholds require more iterations) and performance (lower thresholds increase the possibility of suboptimal assignment). This step is repeated iteratively until all vertices are assigned. For each cluster, the neighbouring vertices not yet assigned to a cluster are selected and their similarity to the neighbouring cluster's seed time-courses is calculated and vertices are again assigned to clusters if their time-course similarity is within a fraction of the maximum such similarity.

\subsection{Clustering the Seed Regions Using a Spatial Constraint}

The number of seeds depends to some extent on the size of the neighbourhoods used to define the original stability map as well as on the amount of smoothing used. The parameters described above led to a smoothness that produced approximately 1000 seeds on each cortical hemisphere, though we also tried less smooth maps, which produced roughly 3000 seeds per hemisphere (final clustering results were marginally worse when using the larger number of seeds).

Whilst there is no 'correct' resolution, the number of seeds produced by the above approach is still much larger than that used typically in functional connectivity studies or expected from a cytoarchitectonic atlas. We thus devised a spatially constrained hierarchical clustering approach to further combine regions hierarchically. The spatial constraint ensured that at each resolution in the cluster tree, the parcellation consists of spatially connected regions.

We calculated Pearson's correlation between all of the regions time-courses and also defined a neighbourhood structure between clusters. Clusters are then joined iteratively by 1) joining two neighbouring clusters whose similarity was maximal among all neighbouring clusters; 2) updating the similarity profile for 
the joint clusters and their neighbours using a linkage rule. We use the linkage function proposed by Ward [13, even though Ward's method assumes Euclidean distances instead of correlations. Importantly, this procedure produces an entire cluster tree and not just a single parcellation. The tree can then be cut at a desired resolution, depending on the application.

\section{Results}

Evaluating parcellation methods is difficult, as no ground truth is available. We thus here look at several necessary conditions a good cortical parcellation should satisfy. The first requirement is that the method should produce similar parcellations when run on different data sets derived from the same subject. We will use this as our main measure of the quality of the parcellation, though it is important to realise that this measure is not sufficient to characterise neuro-anatomically meaningful parcellations. For example a parcellation approach might be biased in terms of the size or shape of parcels it returns and many of these biases would also increase scan-to-scan reproducibility artificially.

\subsection{Scan to Scan Reproducibility}

In our data set we have two sets of 6 10-minute scans acquired from the same subject under identical conditions. We used this data-set to generate two parcellations. To reduce potential variability in the results due to scanning day or order effects, we used the first, third and fifth scan from session one together with the second, fourth and sixth scan from session two to generate the first parcellation. The second parcellation was derived from the remaining six scans.

Similarity between two parcellations was measured using the dice coefficient. Instead of measuring the dice similarity between any two pairs of clusters in two parcellations, we first matched clusters. This was done by matching the two clusters that have the largest dice similarity. This process was repeated (with already matched clusters removed from further comparison). Dice similarities between matched pairs were then averaged to get a scalar measure of parcellation similarity.

The left panel in Figure 1 plots the dice similarity (y-axis) for parcellations obtained by cutting the hierarchical cluster tree at different resolutions ( $\mathrm{x}$-axis). We also show the results we obtained with other approaches in Figure 1. See the caption for details and references.

The only approach which we found to performed similarly to our method was a locally constrained normalised cuts (NCUTS) spectral clustering method where the similarity matrix was constrained to spatial neighbourhoods. However, looking at the parcellations found with this approach (shown in Figure 2(b) , it appears that this method produced clusters that are less anatomically convincing than those derived with our approach (shown in Figure 2(a). In particular, NCUTS completely failed to identify the sensory-motor cortex, which is parcellated into body and face regions with the region growing method. 

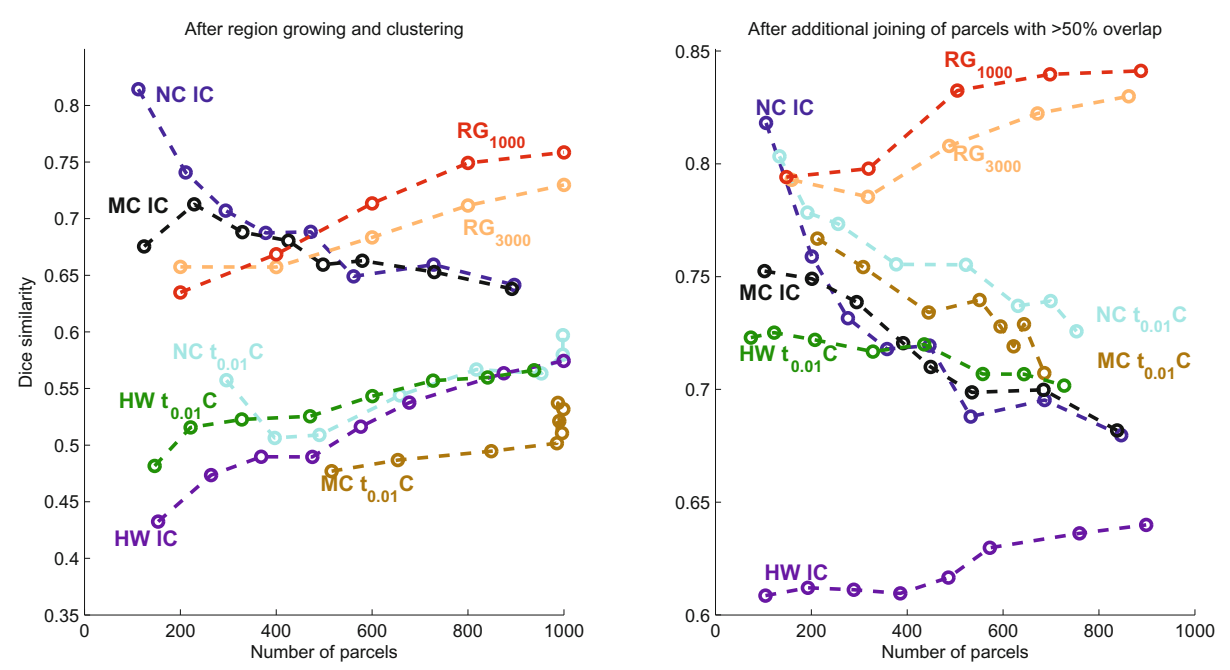

Fig. 1. Dice similarity between matched parcellations vs. parcellation size calculated for parcellations from different datasets of the same subject before (left) and after (right) joining split clusters. Region growing approach with $\sim 1000$ ( $\mathrm{RG}_{1000}$, red) and $\sim 3000$ ( $\mathrm{RG}_{3000}$, orange) seeds outperforms all other tested approaches over a range of parcellation resolutions. Also shown is a small subset of the results obtained with other methods (including the next best performing methods NCUTS with a local correlation similarity measure (NC LC, blue) [4] a spectral clustering approach to optimise network modularity (MC LC, black) 14 and a hierarchical clustering approach using Ward's linkage rule (HW LC) [13, both again with a locally restricted correlation similarity measure. For comparison, the results obtained by the same approaches with a different similarity matrix (a correlation matrix in which values were thresholded so that only $1 \%$ of the entries in each column/row where non-zero [2]) are also shown ( $\{\mathrm{NC}, \mathrm{MC}$, $\left.\mathrm{HW}\} \mathrm{t}_{0.01} \mathrm{C}\right)$.)

The stark difference between the results obtained with our approach and those found with NCUTS seems to be mainly due to NCUTS being strongly biased towards a parcellation with clusters of similar size (compare the histogram inlays in figure 21). Note that the effect of this bias in NCUTS reduces if the number of parcels increases (hence the dice measure decreases for NCUTS in figure 1 with increasing resolution, whilst dice incases for all other methods).

Due to noise effects, a region is sometimes split into two parcels in one parcellation, whilst in another parcellation the regions have been joined. To account for this effect, when comparing parcellations, we produced matched parcellations in which any two clusters in one parcellation that each overlapped more than $50 \%$ with a cluster in the second parcellation, were joined iteratively. Dice similarity after this joining operation is shown in the right panel of Figure 1, Also note that the parcellations shown in Figure 2 are those found after this joining operation and that colours were matched to ease comparison. 

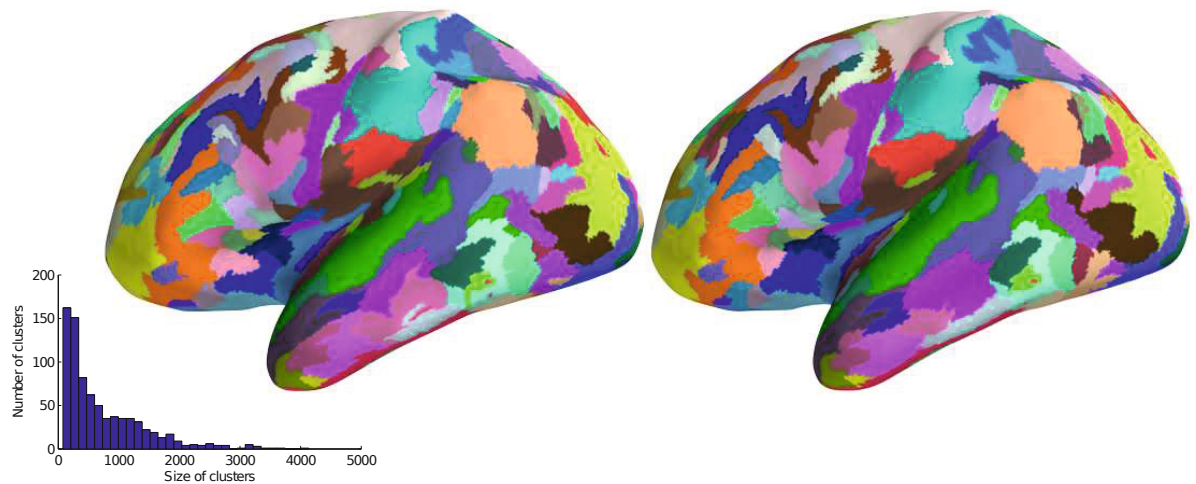

(a) Our region growing approach
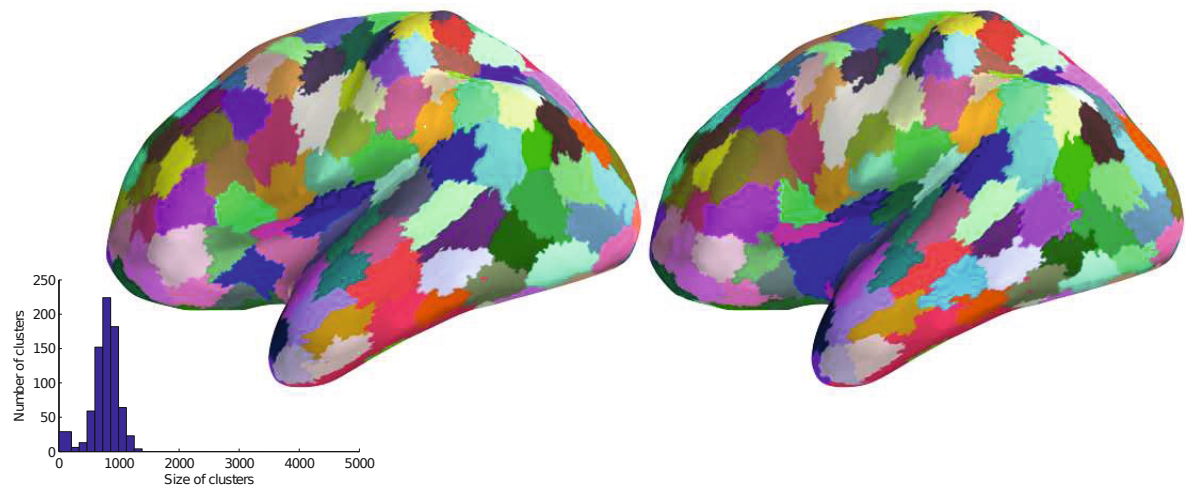

(b) NCUTS with locally constrained correlation [4]

Fig. 2. Same subject parcellation with joint clusters from data-set 1 (left) and dataset 2 (right). Histogram inlays show the distribution of parcel sizes. Parcel colours have been matched to ease comparison.

\subsection{Borders Represent Changes in the Connectivity Profile}

Finally, to show that the borders of the derived parcellations indicate changes in functional connectivity, we drew a path 1 along the cortical surface (shown on the left in Figure 3). Using a different data-set (from the same subject) to that used to derive the parcellation itself. we calculated correlations between the time-series of each vertex along the path (the correlation matrix (thresholded at \pm 0.4 ) is the lower left part of the matrix on the right of Figure (3) and also calculated a connectivity profile feature vector for each vertex along the path (this feature vector was the $\mathrm{r}$ to $\mathrm{z}$ transformed correlation between the vertex time-series and the time-series of 1000 randomly chosen but fixed target vertices on the cortical surface). The correlation between these feature vectors is shown as the upper triangular part of the matrix. Parcellation borders (black lines) align with significant changes in the connectivity profile.

\footnotetext{
${ }^{1}$ The path was chosen so that it roughly crosses parcellation borders at a right angle.
} 

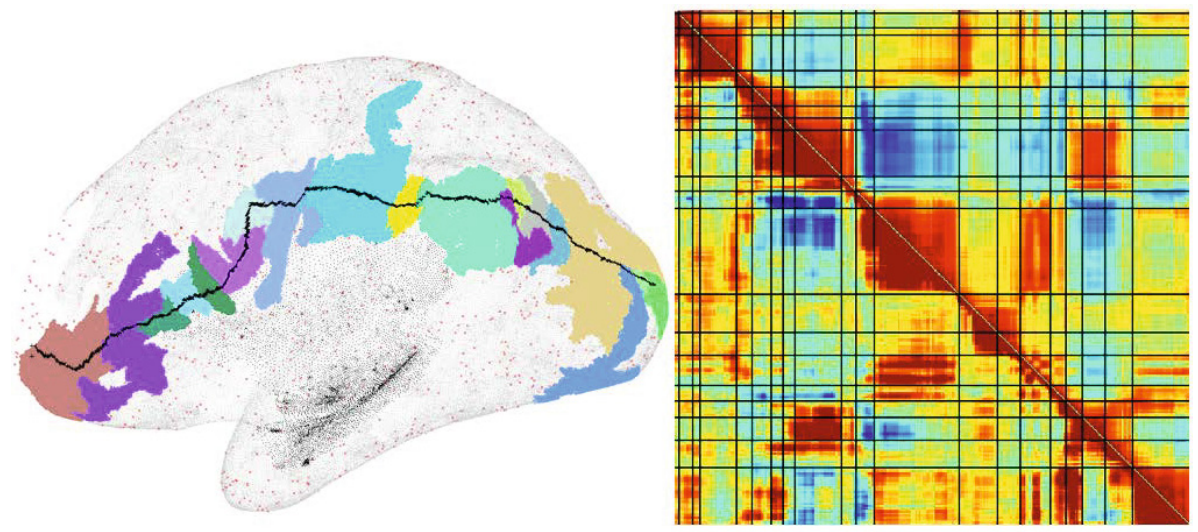

Fig. 3. Path drawn on the cortical surface together with the parcels the path crosses (left) and the correlation (lower left) and connectivity (upper right) profile along the path with parcel borders shown as black lines (right).

\section{Conclusions}

A subject specific cortical parcellation based on resting state functional MRI data has many applications in the study of functional neuro-anatomy and functional brain connectivity. We have proposed an approach that shows high scanto-scan reproducibility and was able to derive parcellation borders that clearly follow changes in the functional connectivity profile. Comparison to other approaches demonstrates the superiority of the proposed approach. Whilst single subject based methods are desirable, they also remain limited in the quality achievable with a single subject's data set and even using 60 minutes of high quality data as done here, parcellations do not yet reach the resolution and reliability desirable for detailed anatomical study. To overcome this, we are currently working on approaches that stabilise single subject parcellations using multi-subject data-sets in a more robust way than is achievable currently with registration based on gross anatomical features.

Acknowledgments. This work was funded in part by the NIH Human Connectome Project 1U54MH091657-01.Data was obtained in the Center for Magnetic Resonance Research (CMRR), University of Minnesota, Minneapolis, MN 55455 using the sequences developed in CMRR 9] with financial support from NIH grants U54MH091657, P41RR008079 and P41EB015894

\section{References}

1. Smith, S.M., Miller, K.L., Salimi-Khorshidi, G., Webster, M., Beckmann, C.F., Nichols, T.E., Ramsey, J.D., Woolrich, M.W.: Network modelling methods for FMRI. NeuroImage 54(2), 875-891 (2011) 
2. Power, J.D., Cohen, A.L., Nelson, S.M., Wig, G.S., Barnes, K.A., Church, J.A., Vogel, A.C., Laumann, T.O., Miezin, F.M., Schlaggar, B.L., Petersen, S.E.: Functional network organization of the human brain. Neuron 72(4), 665-678 (2011)

3. Mumford, J.A., Horvath, S., Oldham, M.C., Langfelder, P., Geschwind, D.H., Poldrack, R.A.: Detecting network modules in fMRI time series: a weighted network analysis approach. Neuroimage 52(4), 1465-1476 (2010)

4. Craddock, R.C., James, G.A., Holtzheimer, P.E., Hu, X.P., Mayberg, H.S.: A whole brain fMRI atlas generated via spatially constrained spectral clustering. Hum. Brain Mapp. (July 2011)

5. Lu, Y., Jiang, T., Zang, Y.: Region growing method for the analysis of functional mri data. NeuroImage 20(1), 455-465 (2003)

6. Bellec, P., Perlbarg, V., Jbabdi, S., Plgrini-Issac, M., Anton, J.L., Doyon, J., Benali, H.: Identification of large-scale networks in the brain using fMRI. NeuroImage 29(4), 1231-1243 (2006)

7. Heller, R., Stanley, D., Yekutieli, D., Rubin, N., Benjamini, Y.: Cluster-based analysis of fmri data. NeuroImage 33(2), 599-608 (2006)

8. Smith, S.M., Miller, K.L., Moeller, S., Xu, J., Auerbach, E.J., Woolrich, M.W., Beckmann, C.F., Jenkinson, M., Andersson, J., Glasser, M.F., Van Essen, D.C., Feinberg, D.A., Yacoub, E.S., Ugurbil, K.: Temporally-independent functional modes of spontaneous brain activity. Proceedings of the National Academy of Sciences 109(8), 3131-3136 (2012)

9. Feinberg, D.A., Moeller, S., Smith, S.M., Auerbach, E., Ramanna, S., Glasser, M.F., Miller, K.L., Ugurbil, K., Yacoub, E.: Multiplexed echo planar imaging for sub-second whole brain fmri and fast diffusion imaging. PLoS ONE 5(12) (December 2010)

10. Smith, S.M., Jenkinson, M., Woolrich, M.W., Beckmann, C.F., Behrens, T.E., Johansen-Berg, H., Bannister, P.R., Luca, M.D., Drobnjak, I., Flitney, D.E., Niazy, R.K., Saunders, J., Vickers, J., Zhang, Y., Stefano, N.D., Brady, J.M., Matthews, P.M.: Advances in functional and structural MR image analysis and implementation as FSL. NeuroImage 23(S1), 208-219 (2004)

11. Beckmann, C., Smith, S.: Probabilistic independent component analysis for functional magnetic resonance imaging. IEEE Transactions on Medical Imaging 23(2), 137-152 (2004)

12. Dale, A.M., Fischl, B., Sereno, M.I.: Cortical surface-based analysis: I. segmentation and surface reconstruction. NeuroImage 9(2), 179-194 (1999)

13. Ward, J.H.J.: Hierarchical grouping to optimize an objective function. Journal of the American Statistical Association 58(301), 236-244 (1963)

14. Newman, M.E.J.: Finding community structure in networks using the eigenvectors of matrices. Phys. Rev. E 74, 036104 (2006) 